\title{
Direct numerical simulation of turbulent flow with an impedance condition
}

\author{
Simone Olivetti ${ }^{1}$, Richard D. Sandberg ${ }^{2}$, Brian J. Tester ${ }^{3}$ \\ Faculty of Engineering and Environment, University of Southampton, SO17 1BJ, U.K.
}

\begin{abstract}
DNS solutions for a pipe/jet configuration are re-computed with the pipe alone to investigate suppression of previously identified internal noise source(s) with an acoustic liner, using a time domain acoustic liner model developed by Tam and Auriault (AIAA Journal, 34, 1996, 917-913). Liner design parameters are chosen to achieve up to $30 \mathrm{~dB}$ attenuation of the broadband pressure field over the pipe length without affecting the velocity field statistics. To understand the effect of the liner on the acoustic and turbulent components of the unsteady wall pressure, an azimuthal/axial Fourier transform is applied and the acoustic and turbulent wavenumber regimes clearly identified. It is found that the spectral component occupying the turbulent wavenumber range is unaffected by the liner whereas the acoustic wavenumber components are strongly attenuated, with individual radial modes evident as each cuts on with increasing Strouhal number.
\end{abstract}

Keywords: Noise reduction, acoustic impedance, jet noise, pipe flows, DNS.

\section{Introduction}

The noise generated by internal flows has recently received attention in the aeronautical field as well as in the industrial field. Noise generated by turbulent pipe flows represents an significant problem for industrial applications such

\footnotetext{
${ }^{1} \mathrm{PhD}$ Student, so2e10@soton.ac.uk

${ }^{2}$ Professor, sandberg@soton.ac.uk

${ }^{3}$ Principal Research Fellow, B.J.Tester@soton.ac.uk
}

Preprint submitted to Journal of Sound and Vibration

July 12, 2014 
as ventilation and piping systems. Furthermore, in the aeronautical field the aircraft's engine is an important source of noise. More specifically turbofan engines are characterized by internal turbulent flows such as by-pass duct flow and nozzle flow through which noise internally generated by the fan, turbine or combustion propagates out to the external observer. Acoustic liners are a common solution to reduce the noise propagating through internal flows. Acoustic liners are passive control devices that convert sound energy into heat through viscous and thermal diffusion processes. They are typically designed as porous surfaces and installed on pipe walls and internal engine ducting walls. Acoustic liners are usually modelled as a mass-spring-damper system and are therefore characterized by a resonance frequency. Previously, researchers had developed mathematical models in order to simulate the performance of acoustic liners (Lansing \& Zorumski [1]). In classical acoustics, where typically no flow is present in the acoustic domain, the liner modelling is quite simple in terms of development and numerical implementation. In contrast, when a viscous flow grazes the acoustic liner surfaces, the mathematical modelling and numerical implementation becomes far more complex (Myers [2]). A grazing flow over an acoustic liner represents a more realistic situation in applications such as ventilation, piping and aero-engines. A number of models have been developed to approximate the steady fluid flow as being uniform, for example, Koch and Mohring [3]; Brazier-Smith and Scott 4]; or Crighton and Oswell [5]; Peake [6]; Abrahams and Wickham [7]; Lucey, Sen and Carpenter [8]. The boundary condition applied at the fluid-solid interface in this case is to match the fluid and solid displacements. This was justified by Eversman and Beckemeyer [9] and Tester [10] by considering the limit of a vanishingly thin inviscid boundary layer at the fluid-solid interface. In this kind of modelling the boundary layer of a grazing flow over an acoustic liner is treated as infinitely thin shear layer on the impedance surface, which is now almost universally applied under the name of the Myers boundary condition (so named because of the work of Myers [2]).

There has been considerable debate over the mathematical and numerical stability of the Myers boundary condition when applied to acoustics over react- 
ing surfaces with slipping flow (for example, Tam and Auriault [11]; Rienstra [12]; Richter and Thiele [13]). Recently there has been growing interest in modelling a finite thickness shear layer such that a no-slip boundary condition can be applied (for example Aurgan, Starobinski and Pagneux [14; Vilenski and Rienstra [15]; Brambley [16]). The difficulty to develop a liner model including a viscous grazing flow might be due the lack of knowledge regarding the physical interaction between the flow field and liner cavities. In order to provide such insight, Tam et al. 17, performed a computational and experimental investigation of the acoustic properties of a three-dimensional acoustic liner with rectangular apertures. It was observed that shed vortices appear on the apertures of the cavities and tend to evolve into rings and align themselves into two regularly spaced vortex trains moving away from the resonator opening in opposite directions. More recently, Zhang and Bodony [18, demonstrated that direct numerical simulation has the potential to provide validated numerical results for acoustic liners with complex geometries. They simulated a locallyreacting honeycomb liner with circular apertures at a variety of sound pressure levels and frequencies. Although computational resources are available to accurately simulate the flow interaction with a single resonating cavity, it is till not possible to extend this analysis to a fully lined wall. Therefore, in order to investigate the effect of acoustic liners on turbulent flows, a CFD solver combined with a time-dependent impedance condition is a possible alternative. Thus, in the current work a time domain impedance model, given by Tam \& Auriault [11] is implemented into an in-house viscous flow solver. The liner model incorporates a frequency independent acoustic resistance and inertance and a cavity reactance inversely proportional to frequency. Acoustic impedance conditions have previously been applied to CFD solvers, see for example Zheng \& Zhuang [19] and Baelmans \& Desmet [20]. However, they used artificial profiles for the boundary layer generating an artificially thickened boundary layer. In consequence, the modelling error due to the large boundary layer thickness leads to a wrong prediction of the NASA flow tube experiment (Watson, Parrot \& Jones [21]). Realistic boundary layers from a CFD simulation were used by Eriksson 
\& Baralon 22]. They showed that a correct prediction of the NASA grazing flow tube experiment could be obtained by using a high-order accurate CFD code. In the present work a DNS solver is used to simulate a fully turbulent subsonic pipe flow. The Tam \& Auriault [11] model has been implemented to simulate the acoustic effect of an acoustic liner on the internally generated noise. The main objective of this study is to investigate the internal noise reduction potential of the liner model and to assess its effect on the turbulent flow. The DNS solver already has been used by Sandberg, Sandham \& Suponitsky [23] for simulations on jet noise which motivated the present work. It is important to state that in the present work the liner is modelled as a uniform, constant, linear impedance surface so there is no need to incorporate a detailed model of the unsteady flow through individual holes unlike Zhang and Bodony [18] and Tam et al. [17. who study a grazing flow over a single and multiple meshed holes.

In this paper the second section introduces the governing equations implemented in the DNS solver. The third section introduces the liner model and its implementation. In the fourth section the Tam \& Auriault [11] model is applied to a fully turbulent subsonic pipe flow. In the fifth section the attenuation capability of a particular liner design is tested and analysed, along with modifications to the inflow perturbations. In the sixth section a wavenumber analysis (equivalent to beamforming) is carried out, in order to separate out the acoustic and hydrodynamic components of the DNS wall pressure and hence more clearly assess the liner performance in suppressing the acoustic noise.

\section{Governing Equations}

The flow under consideration is governed by the full compressible NavierStokes equations. The fluid is assumed to be an ideal gas with constant specific heat coefficients. All quantities are made dimensionless using the nozzle ra-

dius and the bulk velocity within the nozzle. For simplicity, all equations in this section are presented in tensor notation. The non-dimensional continuity, 
momentum and the energy equations are:

$$
\begin{gathered}
\frac{\partial \rho}{\partial t}+\frac{\partial}{\partial x_{k}}\left(\rho u_{k}\right)=0 \\
\frac{\partial}{\partial t}\left(\rho u_{i}\right)+\frac{\partial}{\partial x_{k}}\left[\rho u_{i} u_{k}+p \delta_{i k}-\tau_{i k}\right]=0 \\
\frac{\partial}{\partial t}(\rho E)+\frac{\partial}{\partial x_{k}}\left[\rho u_{k}\left(E+\frac{p}{\rho}\right)+q_{k}-u_{i} \tau_{i k}\right]=0
\end{gathered}
$$

where the total energy is defined as $E=T /\left[\gamma(\gamma-1) M^{2}\right]+0.5 u_{i} u_{i}$. The stress tensor and the heat-flux vector are computed as respectively,

$$
\tau_{i k}=\frac{\mu}{R e}\left(\frac{\partial u_{i}}{\partial x_{k}}+\frac{\partial u_{k}}{\partial x_{i}}-\frac{2}{3} \frac{\partial u_{j}}{\partial x_{j}} \delta_{i k}\right) \quad, \quad q_{k}=\frac{-\mu}{(\gamma-1) M^{2} \operatorname{Pr} \operatorname{Re}} \frac{\partial T}{\partial x_{k}},
$$

where the Prandtl number is assumed to be constant at $\operatorname{Pr}=0.72$, and $\gamma=$ 1.4. The molecular viscosity $\mu$ is computed using Sutherland's law (White, 1991) setting the ratio of the Sutherland constant over freestream temperature to 0.36867 , implying a reference temperature of $300 \mathrm{~K}$. To close the system of equations, the pressure is obtained from the non-dimensional equation of state $p=(\rho T) /\left(\gamma M^{2}\right)$.

It is important to note that the DNS solver is made non-dimensional defining the speed of sound as $c=U / M$, where $U$ is the bulk velocity at the pipe outlet and $M$ the Mach number. Furthermore, length and time is made non-dimensional using $R_{\text {pipe }}$ and $R_{\text {pipe }} / U$, respectively. This ensures the nondimensional consistency of the DNS solver with the acoustic equations shown in the section 3. At the pipe inflow velocity perturbations are calculated using a compressible version of the digital filter technique according to Touber \& Sandham 24], with parameters specified from precursor periodic pipe simulations, and superposed onto mean flow values obtained from the same precursor DNS. At the pipe outlet a zonal non-reflecting characteristic boundary condition is applied in order to avoid spurious reflections ( Sandberg \& Sandham [25]). Details on the length of the pipe needed to achieve fully developed flow and on the 
variation of pressure, density and temperature within the pipe can be found in Sandberg, Suponitsky \& Sandham [23], where it was shown that this approach produces a fully developed turbulent pipe flow from approximately 25-30 radii downstream of the inflow boundary.

\section{Acoustic impedance in the time domain; Tam and Auriault model}

In general, a time-domain representation for a wall-impedance model in the frequency domain is obtained by an inverse Fourier transform of the complex impedance function $Z_{a}(t)$ which leads to the convolution

$$
p(t)=Z_{a}(t) * v(t)
$$

where $p(t)$ is the wall pressure perturbation and $v(t)$ is the wall-normal velocity perturbation component. To obtain the current pressure perturbation at the wall, an integration over the time history of the normal velocity is required. However, the application of this method is limited due to the storage demands and the computational time. In order to overcome this, a time-domain impedance boundary condition based on a mass-spring-damper analogy was first proposed by Tam \& Auriault (1996). It can be written in the form

$$
\frac{\partial p}{\partial t}=R \frac{\partial v}{\partial t}-X_{2} v+X_{1} \frac{\partial^{2} v}{\partial t^{2}} .
$$

In order to obtain Eq. 6 in terms of the wall-normal velocity component, $v$, the linearized energy equation at the wall reduces to $\partial p / \partial t=-\partial v / \partial r$, because of the no-slip condition for the other velocity components. Therefore Eq. 6 becomes

$$
\frac{\partial^{2} v}{\partial t^{2}}=\frac{1}{X_{1}}\left[-\frac{\partial v}{\partial r}-R \frac{\partial v}{\partial t}-X_{2} v\right]
$$

where $R$ is the resistance parameter of a uniform dissipative facing sheet and the two reactance parameters are identified as mass-reactance $X_{1}$ and spring-rate $-X_{2}$, and $r$ is defined as the radial coordinate. In the present formulation, Eq. (7), the reactance is defined as

$$
X=X_{1} \omega-X_{2} / \omega
$$


It should be noted that the parameters $R$ and $X$ are made non-dimensional by $\rho c$, where $\rho$ and $c$ are the mean density and speed of sound, respectively. From Eq. 8 it is possible to define the resonance condition, $X=0$, from which we can define $S t_{\text {resonance }}=\frac{1}{2 \pi} \sqrt{X_{2} / X_{1}} . \quad X_{1}$ and $X_{2}$ have arbitrary values where $X_{1}>0$ and $X_{2}<0$. Since the DNS solver uses a Runge-Kutta scheme for the time marching as explained in Sandberg et al. [26, Eq. (7) is rewritten as a first-order system in time. Let us define

$$
\phi=\frac{\partial v}{\partial t}
$$

thus

$$
\frac{\partial}{\partial t}\left[\begin{array}{l}
v \\
\phi
\end{array}\right]=\left[\frac{1}{X_{1}}\left[-\left(\frac{\partial v}{\partial r}\right)_{W \text { all }}^{\phi}-R \phi-X_{2} v\right]\right] .
$$

It is worth noting that Eq. (7) was originally defined for inviscid 1D models in Tam \& Auriault [11] while in this paper Eq. (7) is being applied to a fully viscous 3D simulation. In the original formulation by Tam \& Auriault [1] the pressure time derivative was replaced by the normal gradient of the normal velocity using the linearized energy equation. This is also possible in the current case because in the DNS a no-slip condition on the wall is applied. Therefore, only the wall-normal component, $v$, is allowed to vary, unlike the other components $u$ and $w$, which are set to zero. As a consequence Eq. (7) is still suitable in the current simulation. It is worth saying that the Tam-Auriault model does not include the hydrodynamic interaction between the boundary layer and the acoustic liner, it is only capable of simulating the acoustic interaction between the liner and the flow field.

\section{Turbulent pipe flow; Lined wall}

In this section a fully turbulent pipe flow is computed using the DNS solver described in Sandberg et al. [26] at $R e=6700$, based on bulk velocity and pipe diameter and $M=0.46$. The pipe with length $L_{\text {pipe }}=50 R_{\text {pipe }}$ is discretized using 624 and 68 points in the axial and radial directions, respectively. The grid 
spacing in the streamwise direction is equidistant with $\Delta z=0.084 R_{\text {pipe }}$. In the radial direction a polynomial stretching is used with maximum and minimum grid spacings $\Delta r=0.026 R_{\text {pipe }}$ and $\Delta r=0.0026 R_{\text {pipe }}$ at the axis $(r=0)$ and wall ( $r=R_{\text {pipe }}$ ), respectively. 64 Fourier modes are used in the azimuthal direction with $100 \%$ de-aliasing, resulting in 130 collocation points in physical space. Figure 1a shows a snapshot of the axial velocity component of the turbulent pipe flow under consideration. Figure $1 \mathrm{~b}$ shows the evolution of the mean axial velocity profile for three different axial positions.

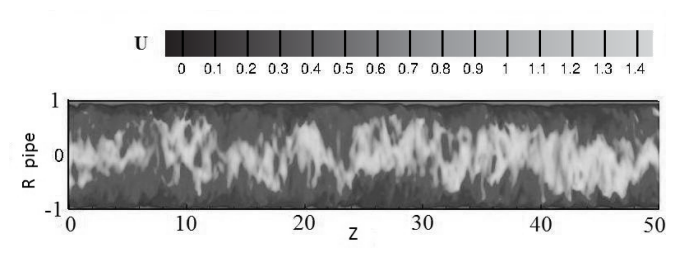

(a)

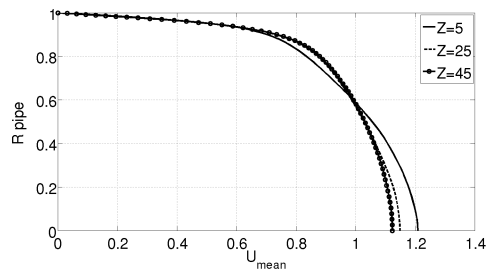

(b)

Figure 1: Turbulent pipe flow, $M=0.46, R e=6700$. a) snapshot of the total axial velocity component; b) axial mean velocity profile for three different axial positions.

Hard wall case vs Lined wall case. The lined-wall case is compared to the hardwall case in order to assess the acoustic attenuation. A validation of the liner model implemented is conducted by injecting into the turbulent flow field sinusoidal signals at the inflow, which propagate through the pipe. The attenuation rates are then compared with eigenvalue solutions from a different code that assumes a uniform mean axial velocity profile. For the liner attenuation studies, the reactance parameters are set so that $S t_{\text {resonance }}=1$ and a sinusoidal input is superposed onto the axial perturbations at the inflow with a frequency of $S t=1$. Figure 2 shows the power spectral density $P S D$ of the pressure at two different locations along the pipe wall. As expected the peak at $S t=1$ is significantly attenuated. Another tone appears at $S t=2$, which is a higher harmonic of the forcing frequency. The amplitude of this tone is also considerably 

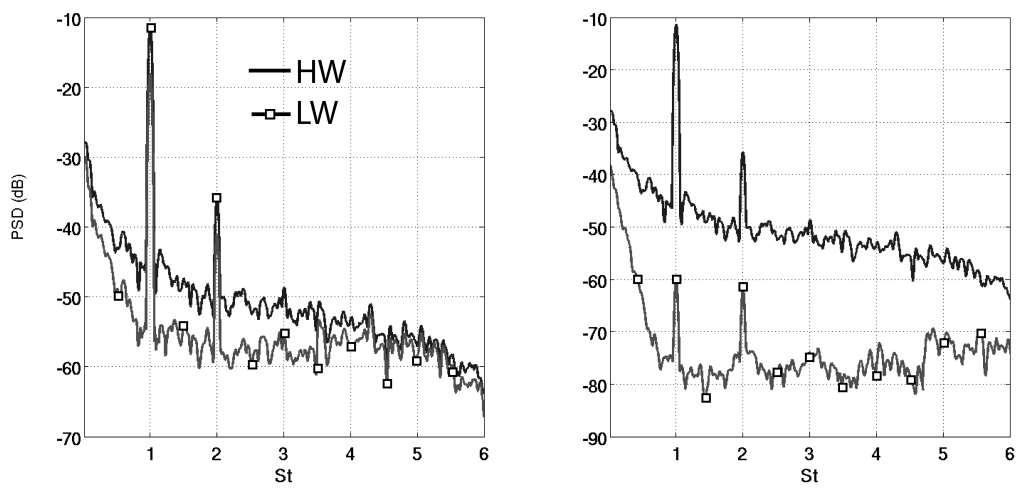

Figure 2: PSD of pressure at $z=2$ (left) and $z=12$ (right) from DNS, hardwall case (solid line), lined wall case (symbols), $R=1$. PSD obtained with Welch method, frequency resolution $\Delta S t=0.1$.

decreased. A reduction of the broad band pressure spectrum is also observed over a wide range of $S t$, and the bandwidth attenuation increases with distance downstream.

Figure $3 \mathrm{a}$ shows the reactance and resistance used for the current pipe case. In order to validate the liner model implementation the wall attenuation at $S t=1$ is computed for different resistance values with the classical eigenvalue solution. Figure $3 \mathrm{~b}$ shows a good agreement between the DNS results and the eigenvalue solution predictions. In order to ensure that the liner model implemented attenuates the acoustic field only, the flow statistics of the lined case is studied and compared to a hard-wall case. In Figure 4 the Reynolds stress components are plotted over the wall distance, $Y^{+}$, calculated at the axial position $z=40$. The normal Reynolds stress components are denoted as $u u, v v, w w$ and $u v$ while the shear stress component is denoted as $u v$. The lined-wall case (squares) is compared to the hard-wall case (solid lines) from the DNS solver and the hard-wall case from a reference DNS in Wu \& Moin [27] (dashed lines). Three zones along the wall distance $Y^{+}$are highlighted. Zone 1, $Y^{+}=0.7-1$ represents a region in the viscous sublayer, Zone $2, Y^{+}=10-19$, is located in the buffer-layer and Zone $3, Y^{+}=40-70$, is located in the 


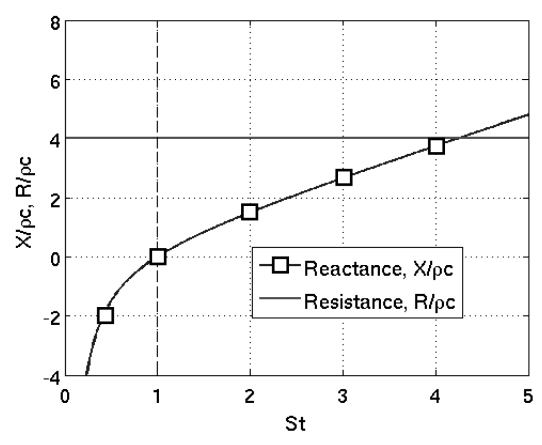

(a)

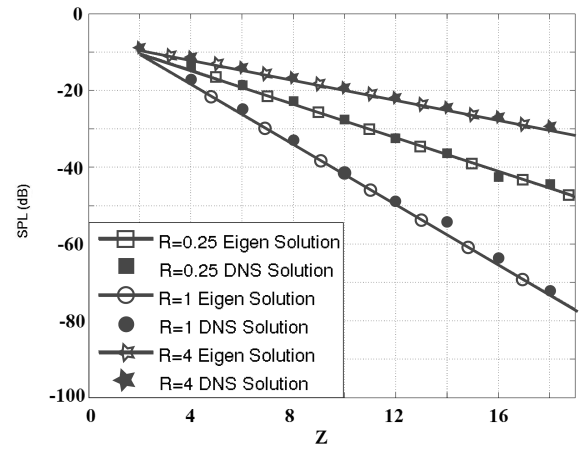

(b)

Figure 3: a) Resistance and reacatance used for acoustic liner, $S t_{\text {resonance }}=$ 1. b) Liner model validation: DNS wall attenuation (symbols), and eigenvalue solutions (lines). Three different values of resistance tested $R=0.25, R=1$ and $R=4$.

logarithmic layer. These zones are investigated in more detail in the following paragraphs.

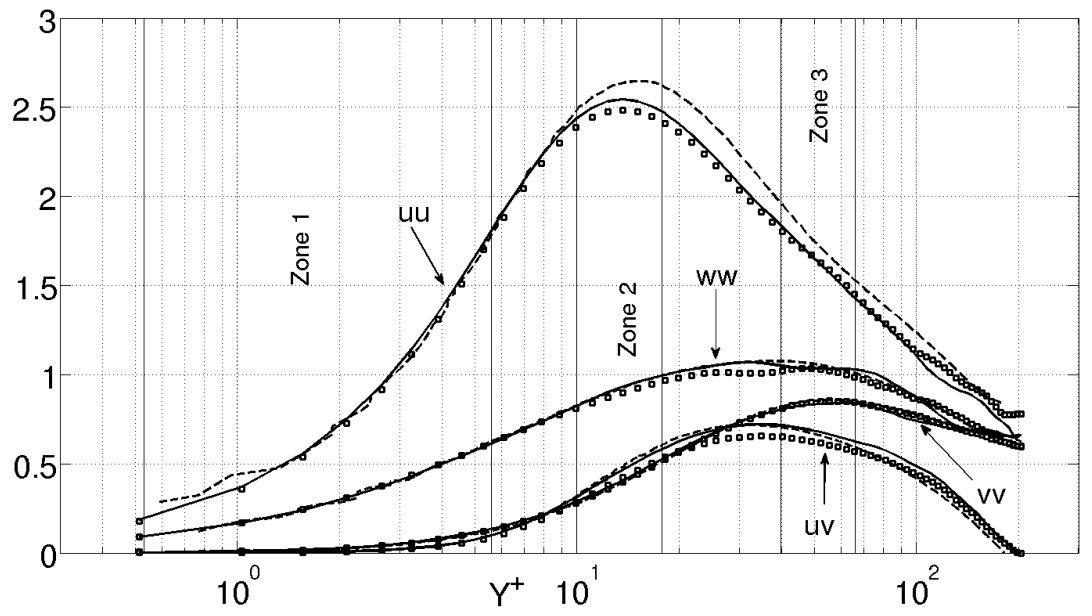

Figure 4: Reynolds stress components; solid lines: DNS hard-wall case; squared symbols: DNS lined-wall case; dashed lines: reference data of Wu $\mathcal{G}$ Moin [27]. 


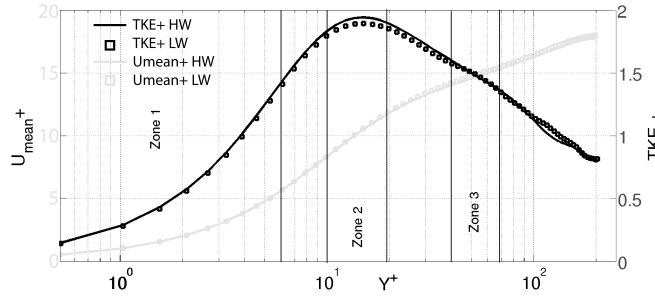

(a)

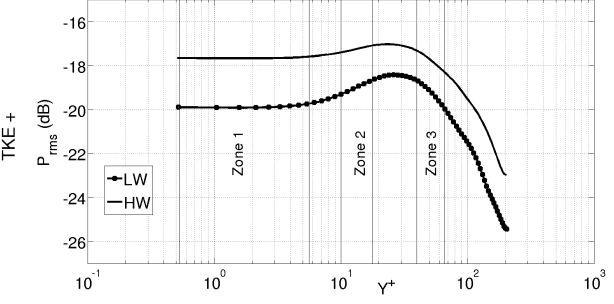

(b)

Figure 5: a) Mean velocity profile $U_{\text {mean }}^{+}$and turbulent kinetic energy $T K E^{+}$; solid lines: DNS hard-wall case; Dots: DNS Lined-wall case. b) Root mean square of pressure over wall distance $Y^{+}$; hard-wall case $(H W)$ vs. lined case $(L W)$.

Figure 4 shows good agreement between the Reynolds stress components from the lined-case and the hard-wall cases, which means that statistically the turbulent flow remains unchanged and only the acoustic field is attenuated. Furthermore, since the DNS results are consistent with the results from Wu \& Moin 27] we can be confident that the turbulent flow has been solved correctly. Figure 5a shows the average axial component $U_{\text {mean }}^{+}$and the turbulent kinetic energy $T K E^{+}$. The lined case is again compared to the hard-wall case showing that the mean velocity profile and the $T K E^{+}$are hardly affected by the liner. The slight reduction of the $T K E^{+}$peaks is due to a small increase in $u_{\tau}$ at the wall. Figure $5 \mathrm{~b}$ shows the comparison of the root mean square pressure, $P_{r m s}$, between the lined and the hard wall case over the radial direction, $Y^{+}$. The overall trend shows a reduction across the boundary layer. Since the turbulent flow remains statistically unchanged the pressure reduction is interpreted as acoustic attenuation only. Figure $5 \mathrm{~b}$ also shows a higher attenuation in the viscous sublayer compared to the other zones. This is due to the presence of the liner which is more effective in the region close to the wall.

The probability density function, $P d f$, of characteristic turbulent quantities such as density, enstrophy, vorticity magnitude and turbulent dissipation rate 
are also studied. This study is carried out in order to confirm the previous results that the liner attenuates the acoustic energy only. These flow parameters are extracted from the three zones highlighted in Figure 4. The time series used to calculate the $P d f$ consist of 500,000 time-steps. Figure 6 shows the $P d f$ of density in the three zones for the lined and hard wall cases. It is clear that in the lined case the $P d f$ peaks at lower values, especially in zones one and two, while in zone three the lined case tends to coincide with the hard wall case. This is due to the influence of the liner which is more effective in the region close to the wall. Figures 7, 8 and 9 show the pdf of enstrophy, vorticity magnitude and turbulent dissipation rate respectively. As expected these quantities are the same for the lined and wall case in all three zones, providing evidence that the liner boundary condition does not affect the turbulent flow.

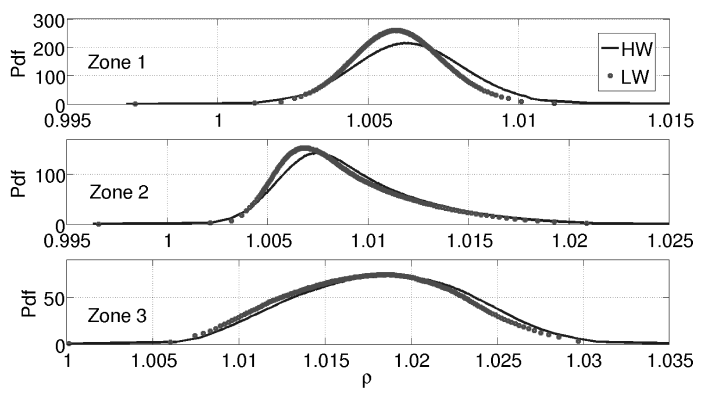

Figure 6: Pdf of density in three different zones along the wall distance $Y^{+}$.
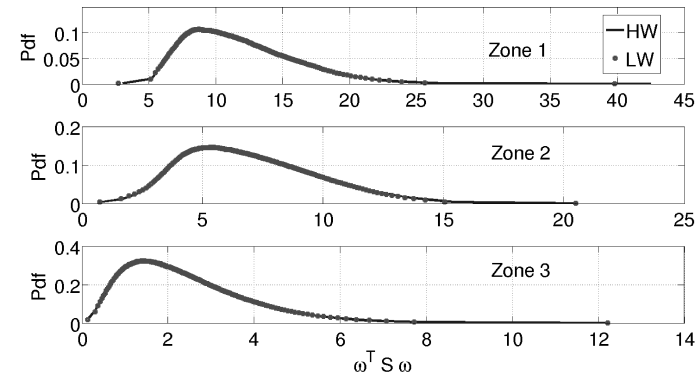

Figure 7: Pdf of enstrophy in three different zones along the wall distance $Y^{+}$. 

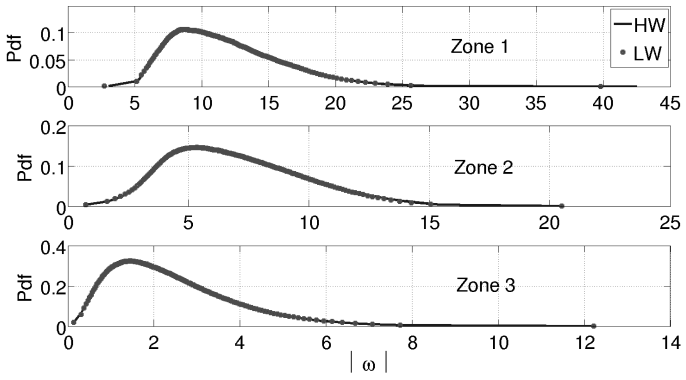

Figure 8: Pdf of vorticity magnitude in three different zones along the wall distance $Y^{+}$.

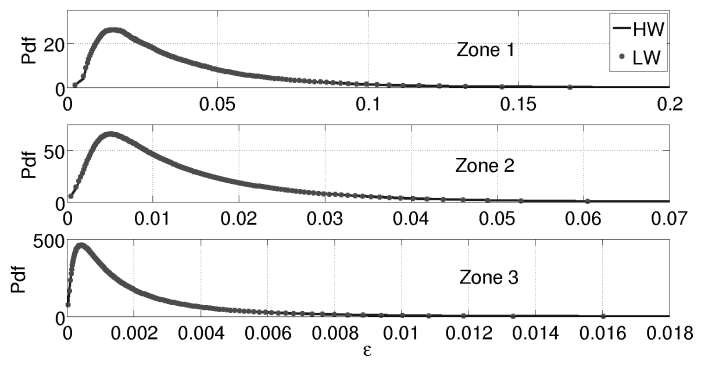

Figure 9: Pdf of turbulent kinetic energy dissipation in three different zones along the wall distance $Y^{+}$.

\section{Liner attenuation; Inlet flow manipulation}

Figure 10 shows the pressure $P S D$ attenuation at the wall for the axisymmetric and the first azimuthal Fourier mode in the frequency domain over the axial positions $Z$. Figure 10 a shows clearly that the peak of the attenuation occurs at the resonance frequency at which the liner has been set, $S t_{\text {resonance }}=1$. The attenuation tends to increase in the downstream direction up to $13 d B$. Figure $10 \mathrm{~b}$ shows the pressure $P S D$ attenuation for the first azimuthal mode. The peak of the attenuation still occurs at $S t_{\text {resonance }}=1$ above the cut on frequency, defined as $S t_{c u t o n}=\alpha_{m n} \sqrt{1-M^{2}} / 2 \pi M$, where $\alpha_{m n}$ are the zeros of the Bessel function (Williams, Crighton \& Dowling [28]). The attenuation is quite low below the $S t_{c u t o n}$ and should actually be zero if the wall pressure 
is purely acoustic. In order to reduce the magnitude of the sound generated by the digital filter technique used at the pipe inlet a manipulation of the inlet condition is attempted. The fluctuating part of the velocity components, $u^{\prime}, v^{\prime}$ and $w^{\prime}$, are set to zero for the axisymmetric mode while $u^{\prime}, v^{\prime}$ and $w^{\prime}$ are left untouched for higher azimuthal modes. Therefore, a new turbulent pipe case is computed keeping the original numerical set up, as described in section 4

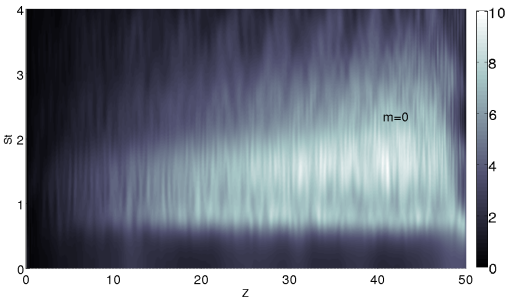

(a)

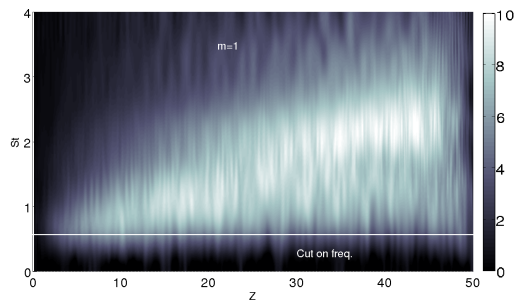

(b)

Figure 10: Wall attenuation (dB) for two azimuthal modes; white line denotes cut on frequency. a) axisymmetric mode. b) Azimuthal mode 1.

Figure 11 shows the pressure $P S D$ attenuation at the wall over the axial coordinate $Z$ of the new turbulent pipe case. The axisymmetric mode shows a modest attenuation in the high frequencies, $S t>2.5$, while the first mode shows a broader attenuation above the cut on frequency. For the first azimuthal mode, an attenuation up to $30 d B$ can be observed, which is the main goal for this application. The liner attenuation is increasingly more effective with distance downstream, although for mode $m=1$ at a fixed $S t$ the attenuation reaches a maximum and then reduces. This may be due to additional noise being generated by the turbulent pipe flow. In the actual simulation the liner is set to resonate at $S t_{\text {resonance }}=1$.

Figure 11a shows no attenuation around $S t=1$, from which we deduce that here the acoustic field is dominated by the turbulent pressure field. This is due to the absence of noise in the low frequency range for mode zero. In contrast, mode 1 shows a modest attenuation around $S t_{\text {resonance }}=1$ where the 


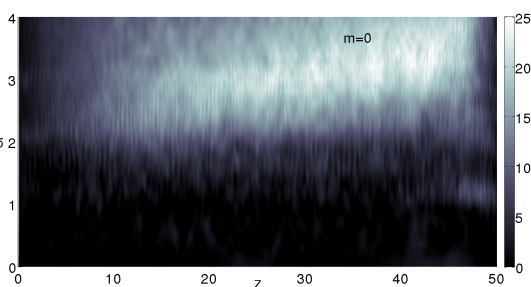

(a)

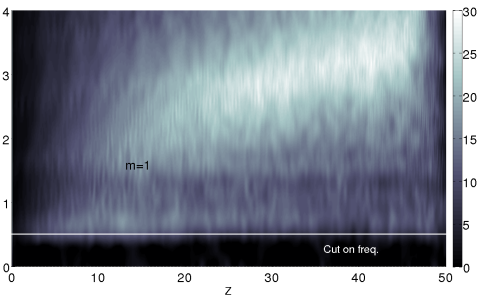

(b)

Figure 11: Wall attenuation (dB) for two azimuthal modes for the new turbulent pipe case; white line denotes cut on frequency. a) Azimuthal mode 0. b) Azimuthal mode 1.

cut-on broad band noise is more significant. Towards the outlet, $z>45$, the attenuation seems to disappear altogether, which is due to the effect of the zonal non-reflecting characteristic boundary condition applied in this region, that is, this region is characterized by non-physical flow and should be ignored.

\section{Wavenumber spectrum analysis}

In order to have a better understanding of how the Tam and Auriault model interacts with the flow field it is useful to discriminate the turbulent field from the acoustic one. In order to do so a Beamformer post-processing method has been applied. This method consists of computing the cross power spectral density $(C P S D)$ of DNS variables such as pressure and velocity in the streamwise direction and calculating its axial wavenumber transform to obtain a $P S D$ wavenumber spectrum, using the following expression

$$
P S D\left(K_{z}, S t\right)=\frac{1}{N^{2}} \sum_{l=0}^{N-1} \sum_{i=0}^{N-1} w_{l}\left(K_{z}\right)^{\prime} C P S D\left[q\left(z_{l}, S t\right), q\left(z_{i}, S t\right)\right] w_{m}\left(K_{z}\right)
$$

where $K_{z}$ is the axial wavenumber, $N$ is the number of grid points in the streamwise direction, $q\left(z_{i}, S t\right)$ is a DNS variable at the axial position $z_{i}$, and $w_{l}\left(K_{z}\right)=\exp \left[i 2 \pi S t M z_{l}\left(-M+K_{z}\right) /\left(1-M^{2}\right)\right]$. For this particular calculation $N=300$ and the time series of the pressure and velocity field consists of 30,000 time-steps. 


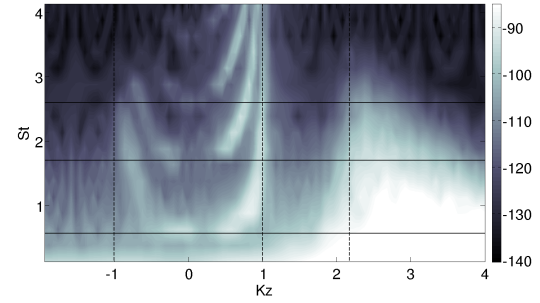

(a)

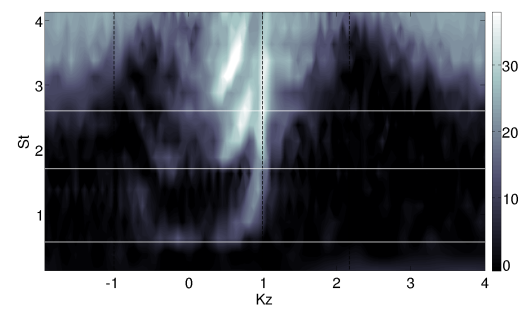

(c)

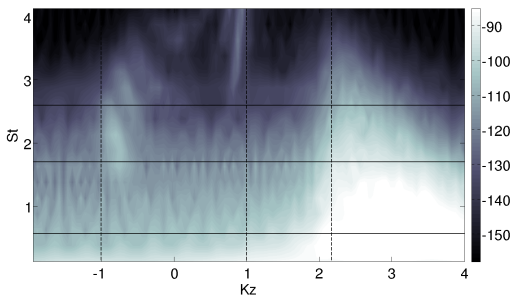

(b)

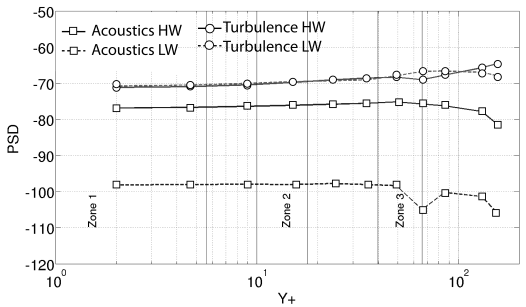

(d)

Figure 12: PSD (dB) of pressure field over wavenumber-frequency domain; horizontal lines cut on frequencies. a) Hard wall case. b) Lined wall case. c) Attenuation (dB). d) Components breakdown of the pressure field. Solid lines with symbols: hard wall case. Dashed lines with symbols: lined wall case.

In Figure 12a the PSD wavenumber spectrum of the wall pressure perturbation is shown. The axial wavenumber range $-1<K_{z}<1$ represents the acoustic field while in the vicinity of $K_{z}=1 / M$ the turbulent field is dominant. In the range $-1<K_{z}<1$, contours of the first, second and third radial modes are visible above their respective cut-on $S t$ (horizontal line). Figure $12 \mathrm{~b}$ shows the $P S D$ wavenumber spectrum when the liner is applied. As expected the acoustic field in the range $-1<K_{z}<1$ is significantly attenuated while the turbulent part remains practically unchanged, as seen more clearly in the attenuation wavenumber spectrum of Figure $12 \mathrm{c}$. It is now possible to integrate the $P S D$ of the pressure field in order to discriminate the acoustic field from the turbulent one for a certain frequency. Therefore, performing the integration of the $P S D$ axial wavenumber spectrum over $-1<K_{z}<1$ and $K_{z}>1 / M$ it 
is possible to plot the trend of the acoustic and turbulent pressure spectra over the wall distance $Y^{+}$, as shown in Figure 12d. We omit turbulent wavenumbers in the range $1<K_{z}<1 / M$ to minimize contamination by the acoustic component. In this case the integration is computed for $S t=1$ since this is the resonance frequency of the liner implemented, see paragraph 4 As expected the turbulent part (circled symbols with solid line) remains unchanged when the liner is present (circled symbols with dashed line). In contrast, the acoustic part (squared symbols with solid line) is attenuated when the liner is applied (squared symbols with dashed line).

It is possible to carry out a similar analysis for the wall-normal velocity component. This gives us a better understanding of how the Tam and Auriault model interacts with the turbulent field. Figure 13 a shows the $P S D$ axial wavenumber spectrum of the $v$ velocity component computed at the wall location $Y^{+}=2$ using Eq. 10. In the acoustic range $-1<K_{z}<1$, contours related to the first, second and third radial modes are visible. Figure $13 \mathrm{~b}$ shows the $P S D$ of the $v$ velocity component over the wavenumber-frequency domain when the liner is present. The acoustic field is attenuated as shown in Figure $13 \mathrm{c}$. Integrating the $P S D$ axial wavenumber spectrum of the wall-normal velocity field over $-1<K_{z}<1$ and $K_{z}>1 / M$, it is possible to plot the trend of acoustic and turbulent velocity spectrum over the wall distance $Y^{+}$as shown in Figure 13d. In this case the integration is computed choosing $S t=3$, since the highest attenuation occurs at this particular frequency, see Figure 13c It is interesting to see how the acoustic part prevails over the hydrodynamic part in the vicinity of the wall for the hard wall case (solid lines with symbols). As expected both the acoustic and turbulent parts, in $(d B)$, tend to minus infinity as the wall is approached, since the hard wall is an impermeable surface. In the lined case (dashed lines with symbols) the turbulent part remains unchanged while the acoustic part has a lower magnitude. In the vicinity of the wall, $Y^{+}=0.5$, the acoustic part clearly prevails over the turbulent part. This result leads us to the conclusion that the acoustic content dominates over the turbulent part when the flow approaches the wall. For this reason the Tam and Auriault model is es- 
sentially coupled to the acoustic component of the DNS field and the turbulent component remains unchanged by the liner model.

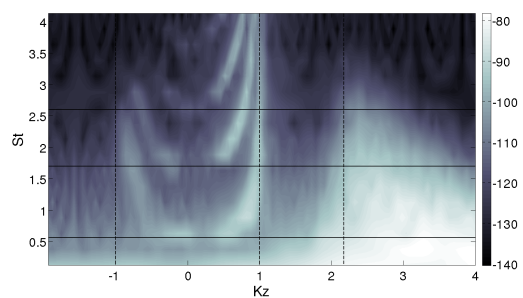

(a)

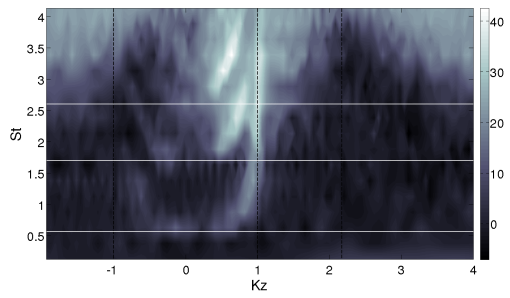

(c)

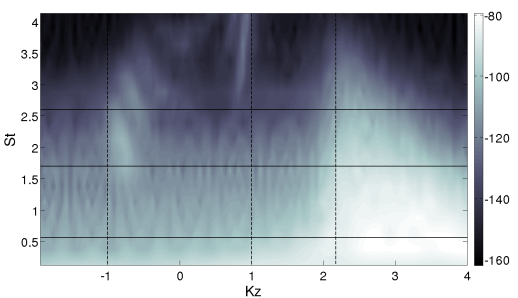

(b)

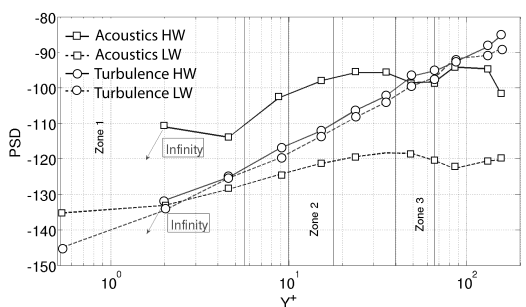

(d)

Figure 13: $P S D(d B)$ axial wavenumber spectrum of the velocity v-component field over wave-frequency domain; magenta lines cut on frequencies. a) Hard wall case. b) Lined wall case. c) Attenuation (dB). d) Components breakdown of the $v$ velocity component field. Solid lines with symbols: hard wall case. Dashed lines with symbols: lined wall case.

\section{Conclusion}

Internally generated pipe noise, previously identified in DNS solutions for a pipe/jet configuration, can be effectively suppressed by replacing the hard-wall boundary condition on the internal pipe wall with a locally reacting impedance boundary condition in the time domain. This has been demonstrated using the Tam \& Auriault [1] model, with suitably chosen liner resistance and reactance parameters. The unsteady pressure field has been attenuated by up to $30 \mathrm{~dB}$ without affecting the velocity field statistics. Application of an azimuthal/axial 
Fourier transform to the wall pressure has shown that the turbulent pressure component is unchanged in the presence of the liner but the acoustic pressure field is strongly attenuated. This is due to the fact that in the viscous sub-layer fluctuations due to the acoustic field dominate over those of the turbulent field and hence the normal velocity of the liner model only couples to the acoustic velocity component of the DNS field. Future work will include an implementation of this liner into a fully turbulent pipe/jet flow to study the effect of the liner on the acoustic far-field.

\section{Acknowledgements}

The first author acknowledges financial support from the Faculty of Engineering and the Environment. We are grateful to Patrick Bechlars for providing us with the tools to obtain probability density functions.

\section{References}

[1] D. Lansing, W. Zorumski, Effects of wall admittance changes on duct transmission and radiation of sound, Journal of Sound and Vibration Vol. 27 (1) (1973) 85-100.

[2] M. K. Myers, On the acoustic boundary condition in the presence of flow, Journal of Sound and Vibration 71 (8) (1981) 429-434.

[3] W. Koch, W. Mohring, Eigensolutions for liners in uniform mean flow ducts, AIAA Journal 21 (2) (1982) 200-213.

[4] P. R. Brazier-Smith, J. F. Scott, Stability of fluid flow in the presence of a compliant surface, Wave Motion 6 (1984) 547-560.

[5] D. G. Crighton, J. E. Oswell, Response of an elastic plate to localized excitation, Phil. Trans. R. Soc. Lond. A 335 (1991) 557-592.

[6] N. Peake, On the behaviour of a fluid-loaded cylindrical shell with mean flow, J. Fluid Mech. 338 (1997) 557-592. 
[7] I. D. Abrahams, G. R. Wickham, On transient oscillations of plates in moving fluids, Wave Motion 33 (2001) 7-23.

[8] S. P. K. Lucey, A. D., P. W. Carpenter, Excitation and evolution of waves on an inhomogeneous flexible wall in a mean flow, J. Fluids Struct. 18 (2003) 251-267.

[9] W. Eversman, R. J. Beckemeyer, Transmission of sound in ducts with thin shear layers - convergence to the uniform flow case, J. Acoust. Soc. Am. 52 (1972) 216-220.

[10] B. J. Tester, Some aspects of sound attenuation in lined ducts containing inviscid mean flows with boundary layers, Journal of Sound and Vibration 28 (1973) 217-245.

[11] C. K. Tam, L. Auriault, Time-domain impedance boundary conditions for computational aeroacoustics, AIAA Journal 34 (1996) 917-913.

[12] S. W. Rienstra, A classification of duct modes based on surface waves, Wave Motion 37 (2003) 119-135.

[13] C. Richter, F. H. Thiele, The stability of time explicit impedance models, AIAA paper 37.

[14] R. M. Baelmans, Y., W. Desmet, Influence of grazing flow and dissipation effects on the acoustic boundary conditions at a lined wall, J. Acoust. Soc. Am. 109 (2001) 59-65.

[15] G. G. Vilenski, S. W. Rienstra, On hydrodynamic and acoustic modes in a ducted shear flow with wall lining, J. Fluid Mech. 583 (2007) 45-70.

[16] E. J. Brambley, Fundamental problems with the model of uniform flow over acoustic linings, J. Sound Vib. 322 (2009) 1026-1037.

[17] J. H. J. M. G. W. W. R. Tam, C. K. W., T. Parrott., A computational and experimental study of resonators in three dimensions, AIAA Paper. 
[18] Q. Zhang, D. J. Bodony, Numerical simulation of two-dimensional acoustic liners with high speed grazing flow, AIAA Journal 49 (2011) 365-382.

[19] S. Zheng, M. Zhuang, Verification and validation of time-domain impedance boundary condition in lined ducts, AIAA paper 43 (2005) 306-313.

[20] R. M. Baelmans, Y., W. Desmet, Time-domain impedance formulation suited for broadband simulations, AIAA paper.

[21] P. R. Watson, L., G. Jones, Experimental validation of a two-dimensional shear-flow model for determining acoustic impedance, NASA Technical Paper.

[22] B. M. B. L. Eriksson, M.O., S. Baralon, Validation of a time and frequency domain grazing flow acoustic liner model, AIAA Journal 47 (8) (2008) $1841-1848$.

[23] S. N. Sandberg, R. D., V. Suponitsky, Dns of compressible pipe flow exiting into a coflow, International Journal of Heat and Fluid Flow 35 (2012) 33-44.

[24] E. Touber, N. Sandham, Large-eddy simulation of low-frequency unsteadiness in a turbulent shock-induced separation bubble, Theor. Comp. Fluid Dyn. 23 (2009) 79-107.

[25] R. D. Sandberg, N. Sandham, Nonreflecting zonal characteristic boundary condition for direct numerical simulation of aerodynamic sound, AIAA Journal 44 (2006) 402-405.

[26] S. V. Sandberg, R.D., N. Sandham, Dns of a canonical compressible nozzle flow, Direct and Large-Eddy Simulation VIII, ERCOFTAC series 15, Springer.

[27] X. Wu, P. Moin, A direct simulation study on the mean velocity characteristics in turbulent pipe flow, J. Fluid Mech. (2008) 81-112.

[28] C. A. P. Williams, D. G., J. E. Dowling, Modern method in analytical acoustics: Lecture notes, Springer. 